\title{
CAPÍTULO III \\ DIMENSIONES DE LA RESPONSABILIDAD SOCIAL \\ COMO ESTRATEGIA DE COMPROMISO CORPORATIVO EN LA EDUCACIÓN SUPERIOR
}

\section{Luis Guillermo Molero Suárez}

Doctor en Ciencias Gerenciales. Compensar Unipanamericana Fundación Universitaria Panamericana. Grupo de Investigación en Ingeniería de Sistemas-GIIS. Correo electrónico: 1molero@unipanamericana.edu.co. Avenida (Calle) 32 No. 17-30, Teusaquillo, Bogotá, Colombia.

\section{María Carolina Villarraga Galindo}

Maestría en Administración de Negocios (MBA). Compensar Unipanamericana Fundación Universitaria Panamericana. Grupo de Investigación EGE. Correo electrónico: mcvillarragag@unipanamericana.edu.co. Avenida (Calle) 32 No. 17-30, Teusaquillo, Bogotá, Colombia.

\section{Víctor Inciarte}

Doctor en Ciencias Gerenciales. Universidad Privada Dr. Rafael Belloso Chacín-URBE. Editorial Inver-E-Group. Correo electrónico: inciartev@urbe.edu.ve. Prolongación Circunvalación No. 2 con Av. 16 Guajira, al lado de la Plaza de Toros. Maracaibo, Estado Zulia, Venezuela.

\section{Lisbeth Marina Molero Suárez}

Doctora en Ciencias Gerenciales. Red Internacional de Desarrollo Fronterizo e Integración Latinoamericana (RINDEFIL). Correo electrónico: lisbethmolero@gmail. com. Av. 46 con Calle 108 \#108E-22. Maracaibo, Estado Zulia, Venezuela.

\section{Diana Carolina Rodríguez Agaton}

Técnico Profesional en Procesos Empresariales. Compensar Unipanamericana Fundación. Universitaria Panamericana. Grupo de Investigación EGE. Correo electrónico: dianacrodriguez@unipanamericana.edu.co. Avenida (Calle) 32 No. 17-30, Teusaquillo, Bogotá, Colombia.

\section{Resumen}

La presente investigación tiene como propósito cardinal, analizar la responsabilidad social como una estrategia dinámica de compromiso empresarial en las universidades de gestión privada, generando un vínculo inexorable de responsabilidad ético empresarial en el escenario de la educación superior y las comunidades. La metodología que identifica a la presente investigación se basa en un diseño documental, de tipo bibliográfico a través 
de los autores Bull (2003), Fernandez (2005), Guedez (2006) \& Quintero (2007); como descubrimientos importantes surgen las categorias promoción de la metamorfosis general para conseguir comunidades justas con democracia social, de los diálogos nacionales, del debate académico sobre modelos de sociedad, y ser promotor de alternativas viables para solucionar contrariedades de tipo sociales, económicos, educativos, ecológicos, entre otros. Por lo que se concluye que estas organizaciones cumplen en teoría con estas dimensiones, considerando que cada una se enfrenta particularmente a retos en su desarrollo y definición de estrategias para lograr un balance positivo entre su función y los beneficios que puedan ofrecer a los distintos estratos sociales en la cual se encuentran inmersas.

Palabras Clave: compromiso dimensiones, estrategias, Responsabilidad Social.

\title{
DIMENSIONS OF SOCIAL RESPONSIBILITY AS A CORPORATE COMMITMENT STRATEGY IN HIGHER EDUCATION
}

\begin{abstract}
The purpose of this research is to analyze social responsibility as a dynamic strategy of business commitment in private management universities, generating an inexorable link of ethical business responsibility in the scenario of higher education and communities. The methodology that identifies the present investigation is based on a documentary design, of bibliographic type through the authors Bull (2003), Fernandez (2005), Guedez (2006) and Quintero (2007); as important discoveries, the promotion categories of the general metamorphosis to achieve fair communities with social democracy, national dialogues, academic debate on models of society, and be a promoter of viable alternatives to solve social, economic, educational, ecological contradictions arise, among others. Therefore, it is concluded that these organizations theoretically comply with these dimensions, considering that each one faces particularly challenges in their development and definition of strategies to achieve a positive balance between their function and the benefits they can offer to the different social strata in which they are immersed.
\end{abstract}

Keywords: commitment, dimensions, Social Responsibility, strategies.

\section{Este capítulo es resultado de un proyecto de investigación culminado.}

\section{Introducción}

En la actualidad, los procesos de cambios y transformaciones de índole social, económico, político, cultural y educativo registrado en los diversos escenarios mundiales, han afectado e involucrado a las organizaciones tanto públicas como privadas, sin excluir las universidades pues estas son fuentes de ventajas competitivas, capaces de adaptarse 
a las exigencias de la globalización, por ello deben examinar su estructura organizativa, procesos de trabajo, filosofía de gestión; a fin de estar preparadas estratégica y éticamente para mantenerse estables y proyectarse en la sociedad actual.

De lo anterior se desprende, que dicho proceso ha facilitado la incursión y recuperación de una perspectiva educativa en el escenario social donde no solo las universidades son capaces de generar servicios educativos para obtener resultados económicos, sino convertirse en fuente de influencia de discernimiento para la construcción de una sociedad sostenible y sustentable. Si bien es cierto, en las últimas décadas los procesos educativos han sido marcados por el avance tecnológico dejando rezagado el valor del capital humano y con ello la practicidad ética-social.

En esta misma línea, las universidades han redimensionado las labores del docente y los instrumentos de aprendizaje, asimismo, han pasado de un paradigma tradicional a un proceso de conversión de entes comprometidos con clientes externos e internos a ella, permitiendo abordar la colaboración de los figurantes pedagógicos y los sociales.

Bajo esta concepción, la responsabilidad social esgrime un soporte al ecosistema universitario, donde la misma se ha planeado desde una dirección ética en la que se acoplan lo estratégico entre las universidades y la sociedad. Esta sinergia, genera una responsabilidad estratégica del saber y actuar para sembrar una proporción entre las partes involucradas; enmarcados en la tarea de la educación, aprendizaje, investigación, creatividad e innovación, apoyados en una base filosófica de gestión y valores éticos, donde se ubique a las personas como centro de participación y enseñanza significativa en la dimensión social.

En este respecto, la presente investigación acomete hacer un análisis de la responsabilidad social como una estrategia de compromiso corporativo en las universidades de gestión privada, para el fortalecimiento del compromiso ético empresarial del sector educación con la sociedad.

\section{Fundamentación teórica}

\section{La Responsabilidad Social Universitaria}

Para Molero, Villarraga, Molero, Inciarte y Sosa (2019), en los escenarios actuales se define la Responsabilidad Social como un elemento de suma importancia dentro del contexto empresarial y administrativo, sin dejar por alto al universitario, en virtud, de que en las organizaciones no solo el beneficio económico debe primar en los intereses privados, sino por el contrario, también en la suma de responsabilidades con los talentos humanos, el colectivo y todo su entorno.

En ese sentido, en la última década, el sector universitario se ha visto en la necesidad de plantearse y en algunos casos replantearse cómo rescatar su papel social, pues, esgrime dentro de su razón social, la construcción de conocimientos para los futuros profesionales que deben considerar poseer mayores atributos de equidad, ética y justicia, entre otros.

Es por ello, que estos dos conceptos se han visto envueltos en diversas reflexiones puesto que el carácter ético, moral y formativo deben circular en los mismos espacios al 
considerar que las universidades deben en cierto punto repensar su carácter como agentes aceleradores dentro de la educación y la Responsabilidad Social.

En consecuencia, de acuerdo con Villar (2007), dentro las directrices que la Responsabilidad Social Universitaria otorga a las universidades están:

- Ubica a las universidades hacia una clara conciencia de su misión.

- Posibilita el despliegue de políticas y estrategias adecuadas que de firmeza a sus áreas sustantivas como universidad.

- Propicia una apropiada concepción de la autonomía enfatizando la capacidad de tomar sus propias decisiones responsabilizándose de sus procesos e impactos y a la vez atendiendo a los requerimientos, interpelaciones y necesidades de todos.

- Crea condiciones para una cultura de la observación y escucha propia y externa (stakeholders), que genere diagnóstico, la evaluación de sus procesos, acciones e impactos presentes y esperados, tanto cognitivos como educacionales, sociales y medioambientales.

- Forjar ideales de transparencia y rendición de cuentas a todo su ecosistema.

- Integrar la Responsabilidad Social como parte de su modelo educativo.

Ante estos supuestos y considerando el propósito de la investigación que versa en analizar la responsabilidad social como una estrategia dinámica de compromiso social en las universidades de gestión privada, se debe reflexionar y generar estrategias que confronten esta nueva realidad a los fines de ser más pertinentes y efectivas en la consecusión de sus objetivos socialmente responsables.

\section{Responsabilidad social en el marco del compromiso corporativo de la educación superior}

Para Bull (2003), la responsabilidad social implica un ámbito de acción bajo tres vertientes principales del negocio, que son: ganancia, impacto ambiental e impacto social. Por tanto, la acción estratégica de este tema no solo les compete a las empresas como eje economicista, sino requiere de una acción conjunta entre los diversos actores de la sociedad, es decir, los empleados, clientes y comunidad local.

Sobre la base de las ideas expuestas, el despliegue de la responsabilidad social subraya una estrategia que proyecta positivamente a las organizaciones, en virtud, de que las empresas deben mantener una conducta ética, moral y de respeto por las personas y el medio ambiente idóneas, lo cual se traducirá en una mejor óptica desde el punto de vista del colectivo.

Por otra parte, es impreciso hacer referencia acerca de un tema que en la actualidad está catalogado como novedoso, aunque su usabilidad se deba a juicios valorativos e impuestos por directrices gubernamentales, de allí que sea visto como una modalidad de obligación empresarial y no como compromiso o demanda social. En tal sentido, la Responsabilidad Empresarial en su contexto social, en las últimas décadas está adquiriendo una nueva dimensión producto a los cambios y transformaciones del paradigma organizacional y políticas de estado imperantes en el entorno empresarial. (Ospina, Molero, Lozano, Becerra y Ruíz, 2019). 
En consecuencia, no se puede establecer un criterio conceptual de Responsabilidad Social Empresarial para determinar su fundamento u origen, sin considerar la base conceptual de especialistas en el área, tal como refiere Guédez (2006), el cual sostiene que "la Responsabilidad Social Empresarial es una idea en plena secuencia de transformación, donde todavía está en investigación los criterios lógicos para argumentarse la historia”, (p.70). Al respecto, para determinar las bases de la Responsabilidad Social es fundamental referenciar los postulados que inciden en su concepción doctrinal e historia por los cuales se hace posible establecer los criterios y principios que enmarcan la misma.

De allí que se discrepen del porqué de su fundamentación, como son las ideas de obligación, retribuir, compartir, demanda, contribuir y corresponsabilizarse en el contexto social. Asimismo, las organizaciones en sus estrategias cardinales deben tener mecanismos donde sea visible el compromiso moral a indemnizar a la sociedad, pues, a esta le debe su desarrollo y crecimiento. (Molero, Ospina, Ayala y Lozano, 2019), (citado en Molero, Ospina, Quevedo, Montenegro y Castiblanco, 2019).

\section{Responsabilidad social a partir una perspectiva estratégica}

Para Bateman y Snell, (2005), "la responsabilidad social es la obligación hacia la sociedad asumida por la empresa, la empresa socialmente responsable maximiza sus efectos positivos sobre la sociedad y minimiza los efectos negativos". (p. 173). Por otro lado, argumentan Robbins y Coulter, (2005) que "la responsabilidad social es el deber de una empresa, aparte de los requisitos legales y económicos, de perseguir metas de largo plazo para bien de la sociedad". (p. 103). Asimismo, "la responsabilidad social empresarial aumenta la competitividad de la empresa y las compañías que apoyan el trabajo voluntario de su plantel de empleados que tienen mejor productividad, porque el personal se identifica más con ellas". (Kliksberg, 2004).

Sin embargo, de las reflexiones anteriores se consigue establecer que la responsabilidad social tiene el menester de considerar ciertos referentes ineludibles, donde las innovaciones en tecnología y la globalización deben instituir elementos para mejorar la calidad de vida de sus interesados y lograr de este modo optimizar el bienestar de los individuos adscritos a ella. (Ospina, Molero, Lozano, Becerra, y Ruiz, 2019), (Molero Suarez, Ospina Rodriguez, Ayala Escobar, y Lozano Rojas, 2019).

\section{Estrategias de la responsabilidad social para la generación de ventajas competitivas en la educación superior}

Dentro del marco de las organizaciones competitivas, las estrategias son acciones asumidas para analizar los indicadores de sus fortalezas, compensar las debilidades organizacionales del entorno, considerando la descripción de la realidad circundante; apoyados en el eje direccionador de la filosofía de gestión constituida por la misión, visión y políticas empresariales, sin desvincular el aporte significativo del marco de su plataforma ética desglosada en valores organizacionales compartidos. 
Según la Alianza Social (Venanchan, 2003), la responsabilidad social a partir de los principios documentales expuestos por la Comisión de las Comunidades Europeas, se entiende como una integración voluntaria, por parte de las empresas, quienes manifiestan preocupaciones sociales y medioambientales en sus operaciones comerciales y sus relaciones con sus participantes, siendo estos: accionistas, proveedores, clientes, trabajadores, administraciones y comunidades locales.

De los supuestos anteriores se desprende, que las organizaciones que integran una gestión de Responsabilidad Social contienen un factor diferenciador en el sector donde participan, en consecuencia, esta gestión debe ser parte activa dentro de su plan estratégico que dé garantías a la práctica responsable para generar un desarrollo sostenible.

Tabla 1. Fundamentos de la responsabilidad social empresarial.

\begin{tabular}{ccc}
\hline Por qué & Causas & $\begin{array}{c}\text { Retribuir (lo que se ha recibido) } \\
\text { Compartir (el exceso) } \\
\text { Contribuir (sin esperar nada a cambio) } \\
\text { Corresponsabilidad (asegurar el entorno) }\end{array}$ \\
\hline $\begin{array}{c}\text { Por qué } \\
\text { Del porqué }\end{array}$ & Origen & Ética (estar bien/pro-actividad) \\
\hline Para qué & Propósito & $\begin{array}{c}\text { Identidad (autoestima organizacional) } \\
\text { Imagen (cómo soy percibido) } \\
\text { Reputación (cómo me valoran) } \\
\text { Posicionamiento (cómo me prefieren) }\end{array}$ \\
\hline Para qué & Finalidad & $\begin{array}{c}\text { Capital social (tejido social) } \\
\text { del para qué }\end{array}$ \\
\hline
\end{tabular}

Fuente: Guédez (2006).

Por otra parte, la responsabilidad social en las organizaciones enfatiza una dirección completa de componentes donde se amalgaman los aspectos éticos, morales y ambientales de forma voluntaria, no obstante, existe un cuerpo normativo que le da rigor a ésta en el contexto de las organizaciones y debe basarse en necesidades dentro del marco social donde participa.

Sin embargo, inicialmente la responsabilidad empresarial dentro de la aceptación expuesta solo era indirecta, consistía en crear y reinvertir riqueza. Esta visión fue complementada después con la idea de proteger socialmente a los trabajadores mediante sueldo, condiciones y capacitación que se ofrecían siempre en función del propósito de buscar mejorar la producción. Posteriormente, apareció la idea de la filantropía, que comenzó por ser una iniciativa individual de los empresarios y más adelante, se reconstituyó en la creación de fundaciones de empresarios y en fundaciones de empresas. Esta estrategia de filantropía se sustentaba en la idea de solidaridad y se expresaba en la dádiva sin pretender nada a cambio. 
Como resultado de esta actitud reactiva se generaba cierta dependencia que no reportaba mejoras a mediano plazo. Para compensar tales debilidades, surge el concepto de inversión social, que pretende actuar sobre las causas del problema más que sobre sus manifestaciones, lo cual se apoya en las ideas de cooperación y reciprocidad, donde se pretende ser más proactivo que pasivo. El amplio marco de las complicaciones sociales promueve después nuevos alcances que se proyectan hacia el concepto de sustentabilidad.

Por su parte, la estrategia representa un patrón de respuesta a un comportamiento del entorno al cual se desenvuelve la derivación de estas inquietudes, la cual, ha conducido a que las empresas adopten la condición de ciudadanía corporativa y bajo este concepto asumen el compromiso con los derechos civiles, económicos y sociales de la sociedad. Son corresponsales con toda y por toda la sociedad, en tanto tienen análogas obligaciones a las de cualquier ciudadano de un país, la proyección de este esfuerzo se identifica con la generación de capital social.

Una excelente síntesis de esta secuencia la ofrece Charon Méndez, en su libro Responsabilidad social de empresarios y empresas en Venezuela durante el siglo XX (citado por Guédez, 2006). Partiendo de su síntesis, se elabora en los cuadros 1 y 2 :

\section{Cuadro 1. Secuencia de las estrategias de la responsabilidad social empresarial.}

\begin{tabular}{ccc}
\hline Estrategia & Características & Principio \\
\hline $\begin{array}{c}\text { Máxima riqueza (la copa } \\
\text { rebosada) }\end{array}$ & $\begin{array}{c}\text { Beneficio creciente a accionistas y } \\
\text { consumidores. } \\
\text { Generar empleos y cumplir las leyes. } \\
\text { Esperar impactos sociales derivados de lo } \\
\text { anterior. }\end{array}$ & $\begin{array}{c}\text { Funciones básicas } \\
\text { y responsabilidades } \\
\text { primarias }\end{array}$ \\
$\begin{array}{c}\text { Protección laboral de } \\
\text { trabajadores asociados ("la } \\
\text { cantidad es para la casa") }\end{array}$ & $\begin{array}{c}\text { Privilegia la realidad interna de la } \\
\text { organización. }\end{array}$ & Justicia y equidad \\
& Atender salario, condición y educación. & social \\
\hline $\begin{array}{c}\text { Filantropía del empresario } \\
\text { y filantropía corporativa } \\
\text { (dar sin buscar impacto ni } \\
\text { retorno) }\end{array}$ & $\begin{array}{c}\text { Atender necesidades sociales externas. } \\
\text { Adar por vías financieras y no financieras. } \\
\text { Es remedial, reactiva y asistencialista. }\end{array}$ & $\begin{array}{c}\text { Solidaridad y } \\
\text { Contribución }\end{array}$ \\
\hline
\end{tabular}

Fuente: Guédez (2006). 


\section{Cuadro 2. Secuencia de las estrategias de la responsabilidad social empresarial.}

\begin{tabular}{|c|c|c|}
\hline Estrategia & Características & Principio \\
\hline $\begin{array}{l}\text { Inversión social (equilibrar } \\
\text { el compacto social y retorno } \\
\text { de la investigación) }\end{array}$ & $\begin{array}{l}\text { Actuar más sobre las causas que sobre las } \\
\text { manifestaciones de los problemas. } \\
\text { Fomentar desarrollo social. } \\
\text { Se ejecuta por vía directa y de cogestión. }\end{array}$ & $\begin{array}{l}\text { Cooperación y } \\
\text { reciprocidad }\end{array}$ \\
\hline $\begin{array}{c}\text { Gestión sustentable } \\
\text { (todos somos responsables } \\
\text { por todo) }\end{array}$ & $\begin{array}{c}\text { Preservar el ambiente y minimizar impactos } \\
\text { negativos. } \\
\text { Equilibrar lo económico con lo social y } \\
\text { ambiental. } \\
\text { Compartir responsabilidades y pensar en } \\
\text { generaciones futuras. }\end{array}$ & $\begin{array}{l}\text { Retribución y } \\
\text { previsión }\end{array}$ \\
\hline $\begin{array}{c}\text { Ciudadanía corporativa } \\
\text { (el derecho a tener derecho } \\
\text { proviene del deber de tener } \\
\text { deber) }\end{array}$ & $\begin{array}{c}\text { Dimensionar sociopolíticamente la } \\
\text { participación empresarial. } \\
\text { Generar compromiso con la sociedad. } \\
\text { Asumir condición de autor en la vida de un } \\
\text { país. }\end{array}$ & $\begin{array}{l}\text { Corresponsabilidad } \\
\text { y participación }\end{array}$ \\
\hline
\end{tabular}

Fuente: Guédez (2006).

Resulta importante aclarar que la evolución expuesta no es de carácter eliminatorio, en tanto, ningún aspecto surge como resultado de la eliminación del precedente; pues en una secuencia excluyente, lo que ocurre es una dinámica incluyente en la cual cada avance permite la integración de nuevos adelantos sin que ellos pauten la desaparición de los otros. El hecho de que la tendencia se oriente hacia la ciudadanía corporativa y las alianzas sociales no descartan necesariamente la estrategia de la filantropía ni la de la invención social.

Para Fernández (2005), la táctica de impulso de la Responsabilidad Social Corporativa propuesta en la Comunicación Europea se sostiene en los siguientes principios: reconocimiento de la naturaleza voluntaria de la RSC, prácticas de responsabilidad social creíble y transparente, focalización en las actividades donde interviene la comunidad , enfoque equilibrado y global de la RSC que circunscriba los elementos sociales, económicos y ambientales, así como los intereses del consumidor, atención a las intereses y características de las pequeñas y medianas empresas (PyME), y finalmente, apoyo y coherencia con los acuerdos internacionales existentes (Normas fundamentales del trabajo adoptadas por la OIT.

A manera de resumen, actualmente las organizaciones, sin escapar el sector universitario, han aceptado la necesidad de incorporar en el marco de sus planes corporativos las estrategias para la gestión de la Responsabilidad social, siempre que ella no perturbe los efectos de los objetivos planificados, pues esta busca la retribución de la inversión realizada a las comunidades bajo programas que permitan obtener valor agregado a los objetivos organizacionales.

En ese sentido, para la consecución estratégica de la gestión de la responsabilidad social universitaria, se requiere del control de recursos y la caracterización de sus capacidades distintivas mediante la efectiva interacción entre las unidades involucradas, de cara al apoyo 
de sus actividades y la participación del capital humano como eje motor para el despliegue de conocimientos e ideas. Por lo tanto, se proyecta más que una obligación gubernamental obligatoria que como una organización comprometida con la sociedad.

\section{Metodología}

La investigación se centró metodológicamente dentro del paradigma cualitativo el cual se basa en identificar la naturaleza profunda de las realidades, su estructura dinámica, aquella que da razón plena de su comportamiento y manifestaciones ( Mártinez y Benítez, 2016). En otro respecto, con relación al conocimiento científico surgido como producto de la presente investigación corresponde a la afirmación positiva de los constructos teóricos acerca de la Responsabilidad Social, a través del procedimiento científico, es por ellos, que el conocimiento generado busca explicar causalmente los fenómenos indagados a través de las teorías ya mencionadas, y por tanto se concibe a la razón como medio para otros fines; es decir, una razón instrumental. (Pérez, 2010).

Por otro lado, busca establecer las relaciones o conexiones existentes entre los fenómenos observados, por lo que igualmente se establece que la observación a la que se incurre es preminentemente objetiva, independiente del sujeto que conoce. El investigador permanece neutral ante la realidad estudiada, con el fin de establecer las relaciones constantes que existen en el fenómeno estudiado, permitiendo prever racionalmente su comportamiento futuro. (Ortecho, 2007).

Se diseñó un estudio documental de tipo bibliográfico, considerando las categorías principales, la Responsabilidad Social Corporativa, Universidad y Responsabilidad, estrategias de responsabilidad, las cuales sirvieron para estructurar el análisis hermenéutico posterior para la definición de nuevas categorías que aseguraron la emergencia de constructos con los cuales incorporar innovaciones a las teorías existentes. (Tamayo y Tamayo, 2006).

\section{Análisis de los resultados}

En la actualidad, es menester de las universidades de gestión privada, considerar todos los aspectos para integrar a la Responsabilidad Social como un compromiso más allá de todo interés, a los fines, de enriquecer desde la academia a todos sus interesados; estudiantes, talentos humanos y sociedad.

Desde la última década, se han venido desarrollando nuevos términos asociados a innovadores medios gerenciales que no solo involucran el despliegue de capacidades, nuevos modelos de negocios productivos, abaratamiento de los costos de manufactura y distribución sino también, el ser sostenibles y sustentables en el tiempo, lo cual, trascenderá en el desempeño necesario de valores éticos y filosofía de gestión.

Se indica asimismo, que en torno a estas ideas, la responsabilidad social implica un fenómeno de interés, tal y como lo expresa Ospina, Molero, Lozano, Becerra y Ruiz (2019), quienes afirman que la responsabilidad social, robustece el ecosistema empresarial, reafirma su identidad corporativa, mejora su perfil ante los interesados y permite una 
transformación radical facilitando el ingreso a nuevos nichos de mercado, la mejora de procesos, productividad y el descubrimiento de nuevas líneas de acción e inversión.

Ante estas reflexiones, las universidades de gestión privada deben estar en permanente gestación a los fines de impactar en el ecosistema social, emprendiendo nuevos escenarios desde sus espacios de responsabilidades y compromisos con la comunidad.

Sin embargo, para hacerlo con sentido ético, deberá vincular sus bases en la práctica de una cultura organizacional con sentido de compromiso ético para garantizar la participación individual y colectiva en ella; además de promover el sentido de identidad bajo una perspectiva de integralidad e interdependencia para formar una cultura sana alineada a su filosofía de gestión. Refiere Quintero (2007), que las empresas están replanteando su papel social y asumiendo que además de derechos, tienen la responsabilidad con su personal y el entorno.

Los temas tratados, indican que las universidades deben tener como quehacer principal la enseñanza de valores éticos y morales, con conciencia y espíritu cívico para formar a los futuros profesionales de excelencia, de esta forma, se podrá establecer el perfeccionamiento de la gestión de responsabilidad social como una eficaz estrategia empresarial para la preparación de personas con capacidad de liderar una sociedad justa, humanista y por demás eficiente.

Así pues, en función a lo expuesto la responsabilidad social universitaria debe cumplir un papel protagónico en la incubación de conocimientos en sus actores para asegurar su calidad de vida individual y colectiva, para la cimentación y progreso oportuno, democrático y solidario de la sociedad, contribuyendo con ello a la búsqueda de la excelencia en escenarios futuros, una autorrealización sustentadora, con sentido de compromiso creativo e innovador para el desarrollo efectivo del entorno.

Las organizaciones socialmente responsables y en este caso, las universidades, deben nutrir los caminos de diálogo permanente con los distintos capitales humanos, a los fines de afianzar los conceptos éticos y morales que el entorno demanda, creando suficientes garantías de compromiso en sus espacios, generando mayores ganancias tanto para los empresarios como a sus colaboradores.

Actualmente las universidades están al tanto que las prácticas asociadas a la responsabilidad social favorecen el progreso sostenible y sustentable de las sociedades, situando sus operaciones diarias en su crecimiento económico y en la optimización de su competitividad, pero al mismo tiempo, contribuyendo a la protección del medio ambiente y fomento del bienestar social, circunscribiendo igualmente los intereses de los consumidores. Según Triomphe (2002), "la responsabilidad social debe incluir, una gestión socialmente responsable, beneficiosa no sólo para las empresas, sino para los asalariados y colectividades públicas". (p. 23).

Desde la perspectiva organizacional, Mintzberg, Brian y Quinn (citado por Contreras y Pelekais, 2005), la estrategia corresponde al elemento que determina las metas básicas de una empresa a largo plazo, así como la adopción de cursos de acción y la asignación de los recursos necesarios para alcanzarlas. 
Cabe mencionar, que la filosofía empresarial lleva supuesto la estrategia en el marco que guía lo que ellas hacen desde la perspectiva de lo que se hace, subrayando el alcance, los recursos, la sinergia. Para Contreras y Pelekais (2005), la ventaja competitiva está compuesta por las características únicas que diferencian a una organización del resto de sus competidores.

Por ello, las acciones universitarias como compromiso social debe ser parte consustancial de un plan estratégico corporativo, sopena de ser afectado por consecuencias de alto riesgo. De esa manera, es necesario subrayar que las estrategias en esta materia han obedecido a una secuencia ajustada a la propia evolución del concepto de empresa y asociada a las demandas del entorno.

\section{Conclusiones}

En un contexto global, las universidades deben actuar considerando lineamientos estratégicos apegados a una filosofía de gestión, en el cual se enmarque una tribuna ética perseverante conllevando a un perfil de cultura organizacional sano en el cual descanse la responsabilidad social, a manera de indicador de acciones corporativas, suscitando la metamorfosis social para lograr unas comunidades justas con democracia social. En tal sentido, establecer sus declaraciones, su papel activo en los diálogos nacionales, en el impulso de diálogos sobre temas nacionales, en el debate académico sobre modelos de sociedad, sobre alternativas viables a alguno de los problemas, sociales, económicos, educativos, ecológicos, entre otros. "Para llegar a las comunidades es necesario el trabajo en conjunto: Universidades, Sector Público y Sector privado".

Luego del análisis de las dimensiones de la responsabilidad social que desde la teoría se presentan, se deduce que las universidades privadas cumplen en gran parte con las mismas, aunque siempre enfrentando retos en cuanto al desarrollo y las estrategias puestas en prácticas por las universidades para lograr una correspondencia entre su función y los beneficios que puedan ofrecer a los diferentes niveles de la sociedad en la cual se encuentran inmersas.

En lo que corresponde con los elementos de la responsabilidad social, las universidades privadas en teoría son un motor de desarrollo para la gestión económica y social que conjuntamente están enmarcadas en un orden jurídico que les permite desarrollar sus acciones para promover e impulsar la salida a los problemas y las carencias de la colectividad.

En relación con las estrategias, las universidades deben reforzar e innovar en las estrategias diseñadas para darle respuesta a los diferentes panoramas, social, político, ecológico y cultural que hoy día vive la sociedad para lograr resultados que corresponden a una acción universitaria efectiva y capaz de transformar la realidad de una región que se encuentra inmerso en enormes cambios.

Para lograr una gestión universitaria enmarcada en valores de responsabilidad social es necesario conformar equipos de trabajo interdisciplinarios que, conjuntamente con las autoridades pertinentes, asuman la implementación del enfoque de Responsabilidad Social Universitaria, esto no sería posible sin la generación de los procesos administrativos adecuados como: 
- Una administración refinada de los recursos humanos y económicos

- Un claro predominio social en las áreas sustantivas de las universidades: formación, investigación y extensión.

- Programas de Responsabilidad Social bien definidos.

- Diseminación de objetivos universitarios.

Finalmente, a través de la incubación de estrategias efectivas, se obtienen los resultados esperados que comprenden el cambio social y la innovación de las conductas universitarias acostumbradas.

\section{Referencias bibliográficas}

Bateman, T. y Snell, S. (2005). Administración una ventaja competitiva. México: Editorial Mc Graw Hill Interamericana Editores.

Bull, B. (2003). Corporate social responsability: The norwegian experience. Documento preparado para la iniciativa sobre ética y desarrollo. Banco Interamericano de Desarrollo.

Contreras, A. y Pelekais, C. (2005). Desarrollo de un sistema conceptualizado de franquicias en tintorerias ecológicas. Venezuela: Astro Data, S.A.

Donelly J. Gibson, J. y Ivancevich, J. (2001). Fundamentos de dirección y administración de empresas. Colombia: Editorial Mc Graw Hill.

Fernández, R. (2005). Administración de la Responsabilidad Social Corporativa. España: Editorial Internacional Thomson Ediciones.

Guédez, V. (2006). Ética y práctica de la responsabilidad social empresarial, el aporte de la empresa al capital social. Caracas, Venezuela: Planeta.

Kliksberg, B. (2004). Responsabilidad Social Empresarial en las Américas. Venezuela: Editorial Fondo Cultural del Centro Internacional de Educación y Desarrollo.

Mártinez Ruiz, H. y Benítez Ontiveros, L. (2016). Metodología de la Investigación social I. Mexico: Cengage Learning Editores, S.A.

Molero, L., Villarraga, M., Molero, L., Inciarte, V. y Sosa, A. (2019). Responsabilidad social como factor multidimensional para concientizar a empresarios sobre su importancia e influencia en empresa, gobierno y sociedad. En L. Molero, M. Villarraga, L. Molero Suárez, V. Inciarte \& A. Sosa. Tendencias en la Investigación Universitaria. Una Visión desde Latinoamérica. Volumen VII. Santa Ana de Coro, Venezuela: Fondo Editorial Universitario Servando Garcés de la Universidad Politécnica Territorial de Falcón Alonso Gamero .

Ortecho. (2007). Ciencia y tecnologìa para el hábitat popular. Editorial Granice.

Ospina, P., Molero, L., Lozano, H., Becerra, Y. y Ruiz, J. (2019). Capital intelectual y responsabilidad social en entornos de programación de realidad virtual. 
En P. Ospina, L. Molero, H. Lozano, Y. Becerra y J. Ruiz. Tendencias en la Investigación Universitaria. Una Visión desde Latinoamérica. Volumén VII. Santa Ana de Coro, Venezuela: Fondo Editorial Universitario Servando Garcés de la Universidad Politécnica Territorial de Falcón Alonso Gamero.

Pérez. (2010). Enfoques metodológicos. Editorial La Salle.

Quintero, C. (2007). Un vistazo diferente a la Responsabilidad Social Empresarial. Realidad: Revista de Ciencias Sociales y Humanas, 113, 337-361. doi:https:// doi.org/10.5377/realidad.v0i113.3409.

Robbins, S. y Coulter, M. (2005). Administración. México: Editorial Pearson Education, 8va Edición.

Tamayo y Tamayo, M. (2006). El proceso de la investigación cietífica. México: Limusa.

Triomphe, C. (2002). ¿Socialmente Responsable? En la Responsabilidad social compartida y los códigos de conducta. Catalunya: Fundación Paul Solidariata.

Venanchan. (2003). Estudio Latinoamericano de Responsabilidad Social Empresarial. Alianza Social.

Villar, J. (2007). Universidad Nacional de Río Cuarto. Recuperado el 01 de 11 de 2019, de https://www.unrc.edu.ar/unrc/psc/pdfs/biblio/2.\%20Javier\%20Villar.pdf.

Vives, A. (2003). Corpororate Social Responsability as a Tool for Competitiveness. Banco Interamericano de Desarrollo. 